\title{
Bioactive Proteinaceous Hydrogels from Designed Bi-Functional Building Blocks
}

Ian Wheeldon, Scott Calabrese Barton*, Scott Banta ${ }^{\dagger}$

Department of Chemical Engineering, Columbia University in the City of New York, New York, NY 10027, USA

*Present address: Chemical Engineering and Materials Science, Michigan State University, East Lansing, MI 48824-1226, USA

Address correspondence to S. Banta, email: sbanta@cheme.columbia.edu

\section{Supporting Material}

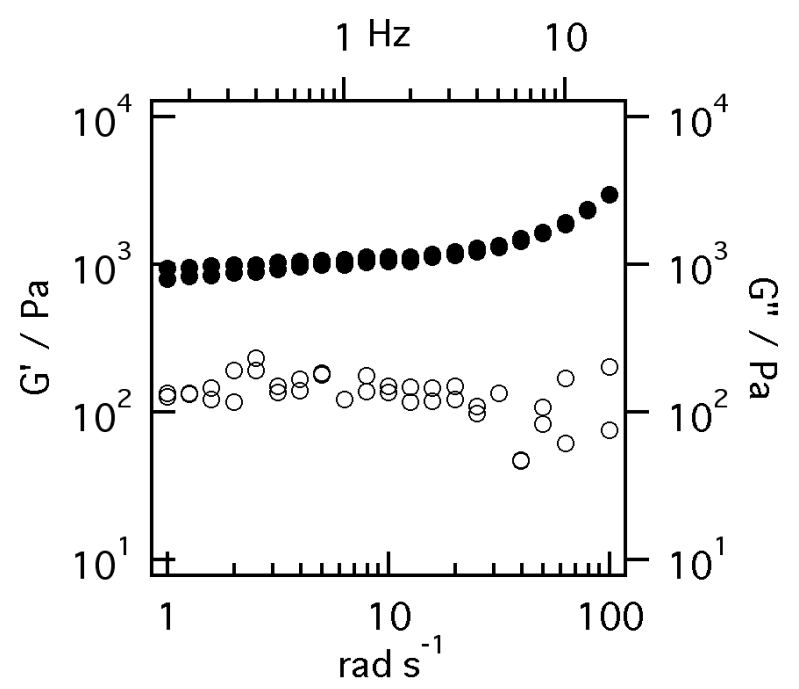

Figure 1 Rheology of $3.4 \mathrm{mM} \mathrm{H-S-ECFP-H.} \mathrm{Frequency} \mathrm{sweep} \mathrm{from} 1$ to 100 to 1 $\mathrm{rad} / \mathrm{s}$ at $1 \%$ strain, $8 \mathrm{~mm}$ stainless steel plate, $500 \mu \mathrm{m}$ gap, $22^{\circ} \mathrm{C}, \mathrm{G}^{\prime}, \bullet$ and $\mathrm{G}^{\prime \prime}$, O. Samples buffered to $\mathrm{pH} 7.0 \pm 0.2$ with $100 \mathrm{mM} \mathrm{Na}$ P. Approximately $30 \mu \mathrm{l}$ of sample was used in the experiment. 


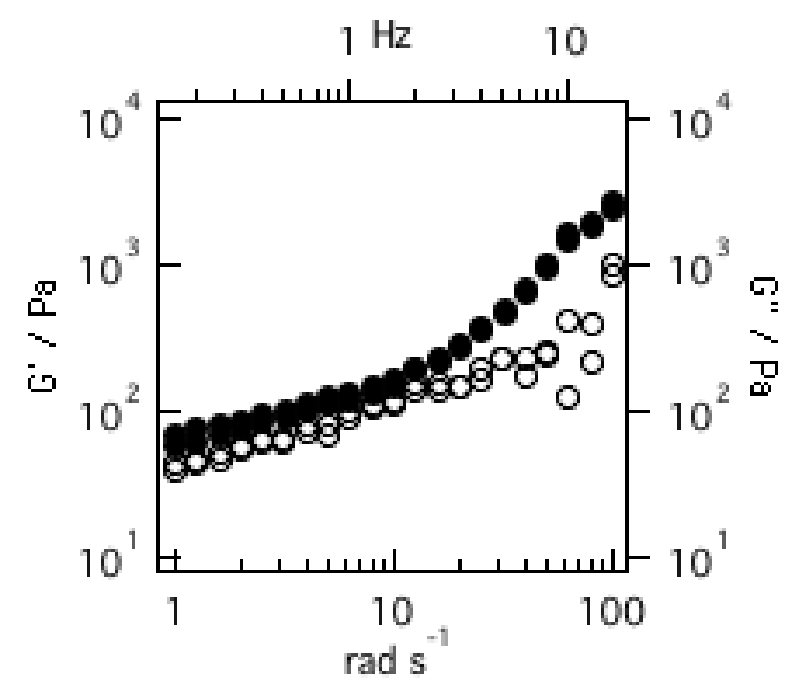

Figure 2 Rheology of 3.4 mM H-S-GFP. Same conditions as stated in SFig. 1, $\mathrm{G}^{\prime}, \bullet$ and $\mathrm{G}^{\prime \prime}, \mathrm{O}$.

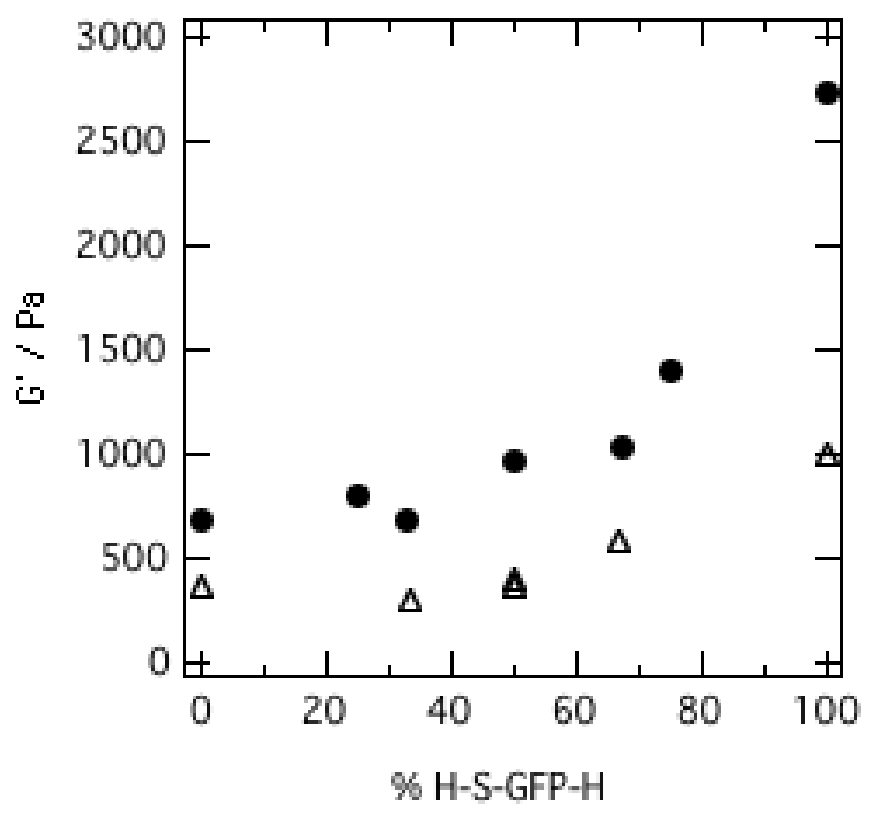

Figure 3 Plateau G' for mixed hydrogel of H-S-GFP-H and H-S-H at constant $\mathrm{H}$ domain concentration of $10 \mathrm{mM} \bullet$ and $6 \mathrm{mM} \triangle$. 

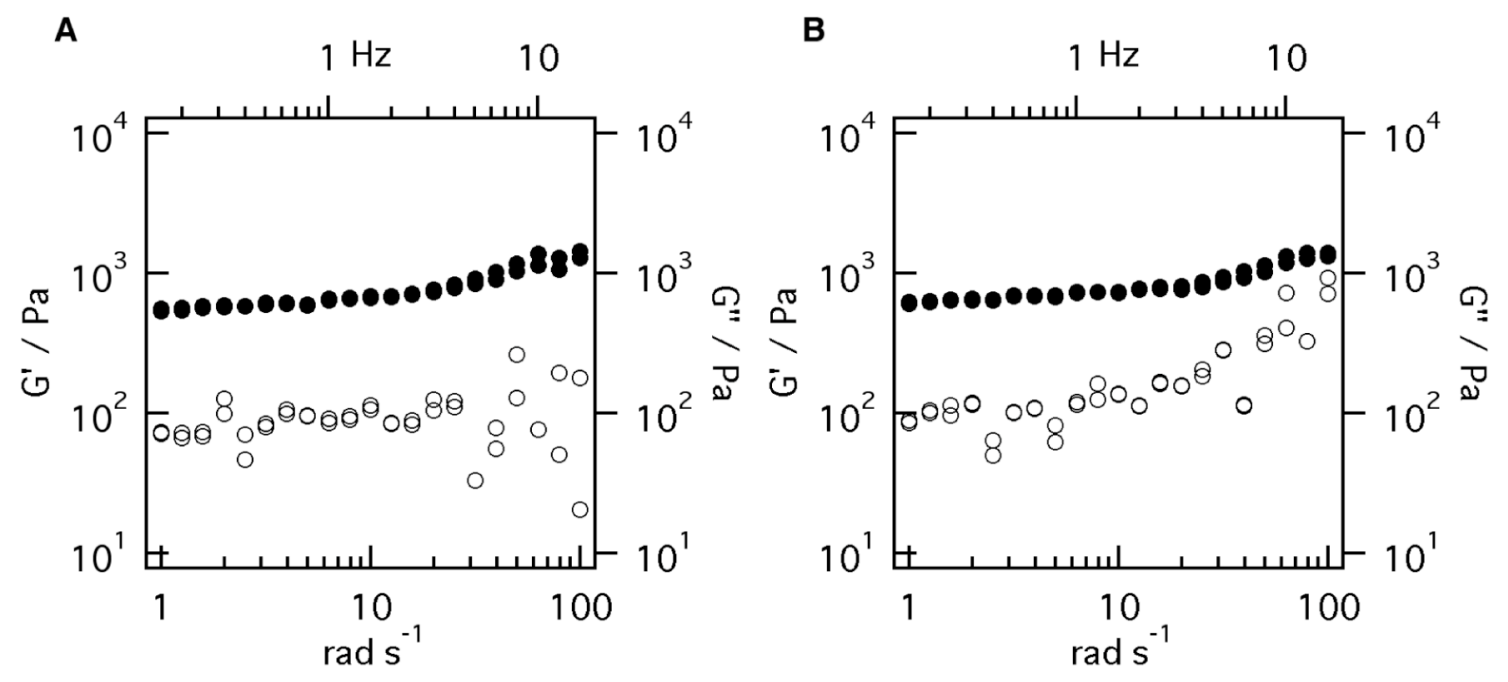

Figure 4 (A) $1.5 \mathrm{mM} \mathrm{H-S-GFP-H} \mathrm{mixed} \mathrm{with} 1.5 \mathrm{mM}$ H-S-DSRED and (B) 1.5 $\mathrm{mM}$ H-S-ECFP-H mixed with $1.5 \mathrm{mM} \mathrm{H-S-DSRED.} \mathrm{Same} \mathrm{conditions} \mathrm{as} \mathrm{stated} \mathrm{in}$ SFig. 1. G',• and $G^{\prime \prime}, ~ O$. 
A.

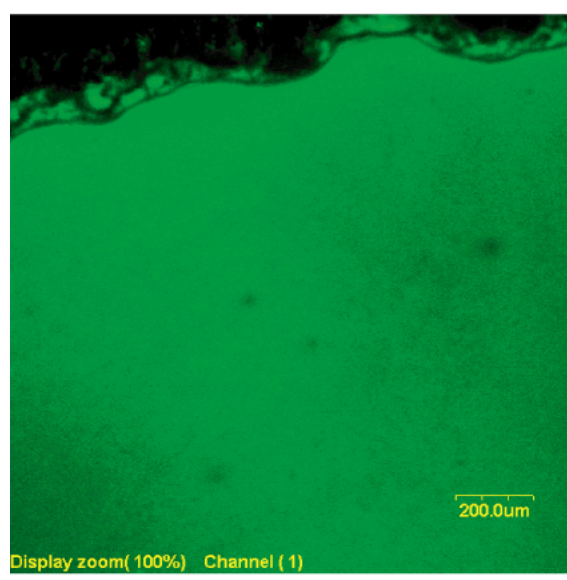

C.

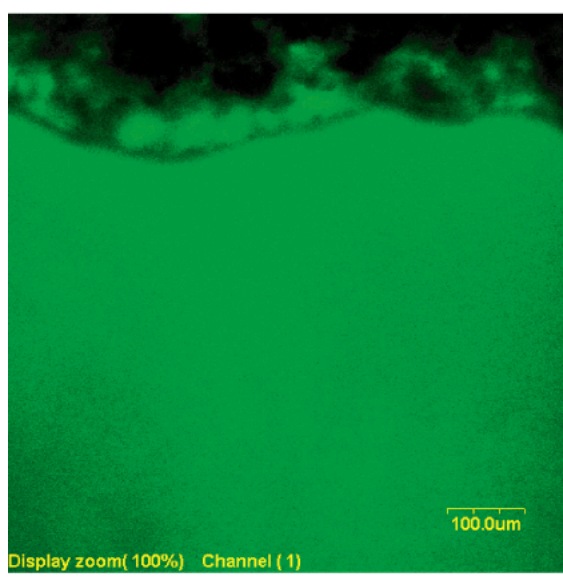

E.

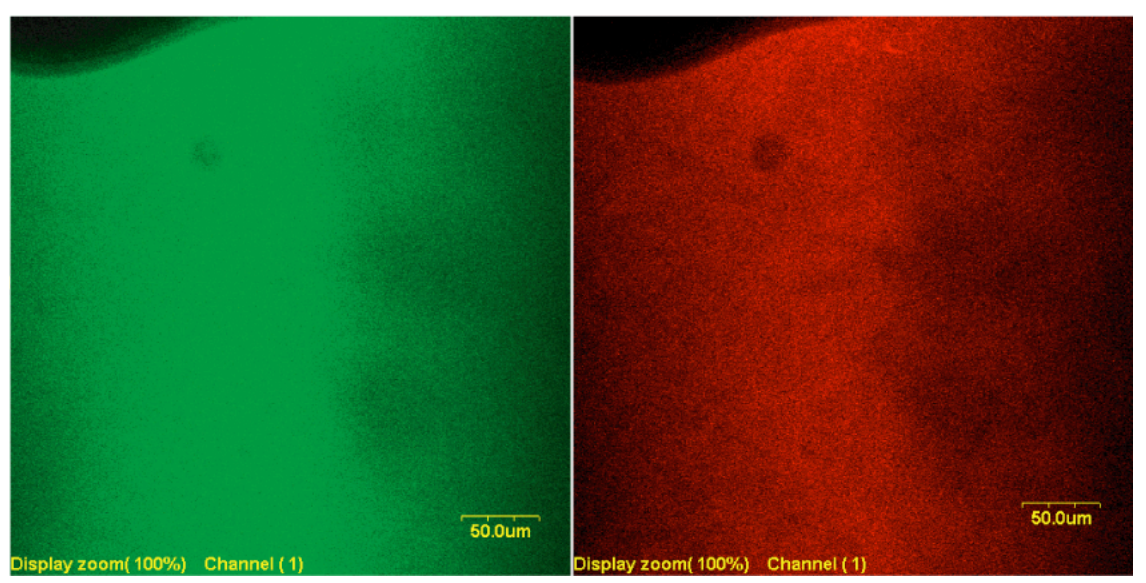

Figure 5 Representative confocal microscopy images of $250 \mu \mathrm{M}$ each of $\mathrm{H}-\mathrm{S}$ ECFP-H and H-S-DSRED in $3.0 \mathrm{mM} \mathrm{H}-\mathrm{S}-\mathrm{H}$. Green and red field images taken separately, (A, C and E) Ex(488nm), $510 \mathrm{~nm} \mathrm{LP}$ and $539 \mathrm{~nm}$ SP filters (B, D and 
F) Ex(568nm) $605 \mathrm{~nm}$ BP filter. (A, B)10x magnification, (C, D) 20x, and (E,F) $40 x$.

Determining FRET efficiency and the Förser radius. FRET efficiency, $D_{F R E T}$, was determined by donor emission quenching (Em. $480 \mathrm{~nm}$ ) in the FRET sample in comparison to the cyan only control sample, equation 1.

$$
D_{F R E T}=1-I_{D A} / I_{D}
$$

Where $I_{D A}$ is the fluorescence intensity of the donor in the presence of the acceptor and $I_{D}$ is the fluorescence intensity of the donor only sample. Fluorophore concentrations were limited to those that within a linear concentration-fluorescence intensity region. FRET efficiency can be related to mean donor-acceptor proximity, $r$, by

$$
D_{\text {FRET }}=1 /\left[1+\left(r / R_{0}\right)^{6}\right]
$$

Where $R_{0}$ is the Förster radius described by

$$
R_{0}=\left(\left(\frac{9000 \ln (10)}{128 \pi^{5} N n^{4}}\right) J(\lambda) Q \kappa^{2}\right)^{1 / 6}
$$

$N$ is Avogadro's number, $n$ is the refractive index of the medium, $Q$ is the quantum yield of the donor, and $\kappa$ is the rotational parameter. The parameter $n$ is taken as 1.4 for solutions of biomacromolecules, $\kappa$ is taken as $2 / 3$ in solution and Q is taken at 0.42 for $\mathrm{ECFP}^{23,24}$. The spectral overlap, $J(\lambda)$, where $\lambda$ is wavelength in $\mathrm{nm}$, is given by

$$
J(\lambda)=\int_{0}^{\infty} I_{D}(\lambda) \varepsilon_{A}(\lambda) \lambda^{4} d \lambda
$$

$\varepsilon(\lambda)$ is the molar extinction coefficient in $\mathrm{M}^{-1} \mathrm{~nm}^{-1}$. Equations 3 and 4 were used to calculate an $\mathrm{R}_{0}$ value of $4.04 \mathrm{~nm}$ for the ECFP and DSRED FRET pair. 